\title{
Vivre sur fond de frontières. Les migrants du Mozambique à Johannesburg
}

Living with borders in the background: migrants from Mozambique in Johannesburg

La vida en el contexto de las fronteras. Los migrantes de Mozambique en

Johanesburgo

Dominique VIDAL

\section{(2) OpenEdition}

\section{Journals}

\section{Édition électronique}

URL : http://journals.openedition.org/conflits/17301

DOI : 10.4000/conflits. 17301

ISSN : $1777-5345$

Éditeur :

CCLS - Centre d'études sur les conflits lilberté et sécurité, L'Harmattan

Édition imprimée

Date de publication : 20 décembre 2008

Pagination : 101-117

ISBN : 1157-966 X

ISSN : 1157-996X

Référence électronique

Dominique VIDAL, "Vivre sur fond de frontières. Les migrants du Mozambique à Johannesburg »,

Cultures \& Conflits [En ligne], 72 | hiver 2008, mis en ligne le 19 mai 2009, consulté le 30 mars 2021.

URL : http://journals.openedition.org/conflits/17301 ; DOI : https://doi.org/10.4000/conflits.17301 


\section{Vivre sur fond de frontières. Les migrants du Mozambique à Johannesburg ${ }^{1}$}

\section{Dominique VIDAL}

Dominique Vidal est professeur de sociologie à l'université Paris-Diderot et chercheurà l'URMIS.

Tétude des migrations contemporaines rencontre la question des frontièLres sous au moins trois angles. Celui, en premier lieu, des frontières étatiques dont la mise en place et le développement ont été analysés comme l'élément politique permettant de distinguer l'immigration moderne d'autres formes de migrations, lorsqu'un migrant franchit une frontière juridique et devient un étranger ${ }^{2}$. Celui, en deuxième lieu, de la recherche urbaine qui montre que les grandes métropoles, tout en constituant plus que jamais des destinations pour les migrants, voient se développer des frontières sociospatiales résultant de l'action de ceux qui cherchent à se protéger de l'altérité 3 . Celui, enfin, des travaux sur l'ethnicité soulignant que des frontières ethniques se construisent dans les relations entre immigrés et populations plus anciennement installées ${ }^{4}$.

Ces différentes problématiques se croisent en Afrique australe au point d'y être parfois inextricablement imbriquées. Il est peu dire en effet que la région offre une large gamme de frontières pour organiser l'espace, régle-

1. La recherche dont ce texte est issu a bénéficié du soutien de l’Institut français d'Afrique du Sud.

2. Zolberg A., "International Migrations in Political Perspectives" in Kritz M.M. et al. (eds.), Global Trends in Migration. Theory and Research on International Population Movements, New York, Center for Migration Studies, 1983, pp. 3-27 ; Noiriel G., La Tyrannie du national. Le droit d'asile en Europe (1793-1993), Paris, Calmann-Lévy, 1991.

3 . Sur la question, et selon des déclinaisons différentes, voir notamment Sassen S., Appiah K.A., Globalization and its Discontents. Essays on the New Mobility of People and Money, New York, New Press, 1999; Bordreuil J.-S., «La ville desserrée » in Body-Gendrot S., Lussault M., Paquot T., (dirs.), La Ville et l'urbain. L'état des savoirs, Paris, La Découverte, 2000, pp. 169-182 ; Maurin E., Le Ghetto français. Enquête sur le séparatisme social, Paris, Le Seuil, 2004.

4 . Poutignat P., Streiff-Fenart J., Théories de l'ethnicité, Paris, PUF, 1995. 
menter les mobilités, catégoriser les populations et déterminer leurs statuts respectifs. Le cas des migrants du Mozambique dans la région de Johannesburg - la plus ancienne et la plus importante des migrations de travail en Afrique du Sud - en est une illustration remarquable 5. Cet article se donne pour objet de montrer comment le rapport à l'espace et la construction identitaire de ces migrants soulèvent la question de la frontière, que celle-ci soit entendue dans un sens spatial, dans une acception juridique ou comme limite traçant les contours d'un groupe ethnique ${ }^{6}$. La polysémie du terme, loin d'être ici un obstacle, rappelle au contraire de manière éclairante qu'une frontière n'est pas seulement une ligne entre deux Etats, mais peut aussi séparer des territoires au sein d'un même espace national et se former dans l'esprit des individus quand ils en viennent à s'identifier à des ensembles distincts. Dans toutes ces situations, les frontières ont pour point commun d'engendrer des effets sociaux et politiques résultant essentiellement de l'action des Etats.

On rappellera pour cela, dans une première partie, l'importance des frontières établies à l'époque coloniale et sous l'apartheid dans la structuration politique du social et les conditions de vie des migrants du Mozambique en Afrique du Sud. On s'intéressera ensuite aux conséquences de la fin de l'apartheid sur la situation des migrants africains dans le pays et l'apparition d'une conceptualisation différente du national et de l'étranger. La dernière partie montrera comment les Mozambicains, confrontés à l'hostilité des Noirs sudafricains, se différencient des autres Africains de Johannesburg, en dressant une frontière ethnique qui concourt au maintien de l'identité personnelle, mais qui ne possède pas une teneur suffisante pour l'action collective.

\section{Des frontières aux conséquences durables}

Comprendre la présence de frontières dans l'expérience des migrants du Mozambique dans le Johannesburg post-apartheid suppose que soit, au préalable, évoquée une série d'éléments de contexte indispensables à tout développement sur la question.

5. Il n'existe pas de données fiables sur le nombre de Mozambicains ou de Sud-Africains d'origine mozambicaine résidant aujourd'hui en Afrique du Sud. Selon les statistiques officielles ou certaines évaluations, les chiffres varient entre 300000 et plus de 2 millions.

6. Cette recherche s'appuie sur des matériaux recueillis, entre 2005 et 2007, au cours de quatre missions d'une durée totale de sept mois, à Johannesburg et à Maputo. Ceux-ci consistent en des entretiens (soixante-dix) avec des migrants et des anciens migrants de sexe masculin, des observations réalisées sur différents sites (lieux de travail, de transit et de résidence principalement), des conversations avec d'autres migrants africains à Johannesburg et des SudAfricains connus dans les occasions les plus diverses, ainsi que des données statistiques issues de l'African Cities Project (ACP) de 2006, une étude longitudinale et comparative des réfugiés, des demandeurs d'asile, des migrants et des non-nationaux de Johannesburg, Lubumbashi (République démocratique du Congo), Maputo (Mozambique) et Nairobi (Kenya). 


\section{Les frontières de l'époque coloniale}

Un des acquis les mieux établis de l'histoire de la colonisation en Afrique réside dans le rôle des Etats coloniaux dans la fabrication des ethnies ${ }^{7}$. Les habitants des territoires actuels de l'Afrique du Sud et du Mozambique n'ont pas échappé à ce processus ${ }^{8}$. Des populations rurales, organisées autour de petites unités où prévalent des relations essentiellement définies par la parenté, sont catégorisées en différentes ethnies à l'époque coloniale. Ces catégorisations ne restent pourtant pas longtemps extérieures à ceux qu'elles concernent, lesquels les reprennent pour se dire et dire autrui. Des catégories comme les «Zoulous », les « Xhosas », les «Tswanas », par exemple, entrent alors dans le lexique des habitants de la région pour devenir des principes cognitifs de l'altérité. La dénomination "Shangaan » rassemble, selon cette logique, une bonne partie des populations du Nord-Ouest de la République sud-africaine et du Sud du Mozambique.

A partir des années 1850, les premiers migrants de ce qui est alors la colonie portugaise du Mozambique arrivent sur le territoire de l'actuelle Afrique du Sud pour travailler dans les plantations de la colonie britannique du Natal 9 . La question du franchissement de cette frontière prend un tour nouveau en 1886, après la découverte de champs aurifères sur les lieux de ce qui devient la ville de Johannesburg. Les mineurs blancs accourus du monde entier ne suffisant pas, le recours à la main-d'œuvre africaine s'impose comme une nécessité. Afin de réguler l'offre de travail et les salaires payés, la Chambre des Mines crée, en 1893, un Département du travail des indigènes qui s'assure la coopération des Portugais pour recruter dans leur colonie du Mozambique et empêcher l'action des recruteurs indépendants. Lisbonne a tôt fait de comprendre son intérêt dans l'exportation et la taxation des revenus des mineurs. Après avoir dominé les chefferies du Sud du Mozambique au terme de la guerre Luso-Gaza (1875-1897), les autorités portugaises imposent aux chefs vaincus de fournir des contingents d'hommes, sous peine d'être eux-mêmes retenus en captivité ${ }^{10}$. Le contrôle étatique de cette migration dès ces premiers temps fait apparaître en conséquence la figure du migrant clandestin à la fin du XIX ${ }^{\mathrm{e}}$ siècle, bien que, avant que ne soit réprimée l'immigration mozambicaine sur le territoire sud-africain, il était question d'empêcher l'émigration clandestine vers l'Afrique du Sud.

Un cadre juridique structure ces migrations pendant près d'un siècle : le «système du travail migrant » (migrant labour system), comme on l'a nommé en

7. Voir à ce propos : Amselle J.-L., M'Bokolo E., Au cour de l'ethnie. Ethnies, tribalisme et Etat en Afrique, Paris, La Découverte, 1985.

8. Harries P., Work, Culture, and Identity. Migrant Laborers in Mozambique and South Africa, c. 1860-1910, Johannesburg, Witwatersrand University Press, 1994 ; Fauvelle-Aymar F.-X., Histoire de l'Afrique du Sud, Paris, Le Seuil, 2006.

9. Harries P., op. cit.

10. Ibid. 
Afrique australe. Il se met en place après l'ouverture des premières mines d'or et s'étend par la suite à tout le secteur minier et à une partie de l'agriculture. Il organise la migration temporaire, pour des périodes de douze à dix-huit mois, de travailleurs africains des zones rurales de l'Afrique du Sud et des pays avoisinants (le Mozambique, le Lesotho, le Swaziland, le Botswana, le Zimbabwe, la Zambie, le Malawi) ${ }^{11}$. Ceux-ci ne reçoivent qu'une partie de leur rémunération sur place et touchent le reste une fois de retour au pays, où ils se réinstallent dans leurs villages dans l'attente d'un nouveau contrat. Cette rotation organisée poursuit un double but. Elle veut, d'une part, empêcher la constitution d'un groupe ouvrier permanent. Elle entend interdire, d'autre part, l'urbanisation des Africains, tenus comme irréductiblement ruraux et n'ayant pour seule raison de se trouver dans les zones industrielles que la satisfaction des besoins en main-d'œuvre des Blancs. C'est dans ce cadre qu'au début du XXe siècle, la Chambre des Mines crée la WENELA (Witwatersrand Native Labour Association) et obtient des Portugais le droit d'installer des bureaux de recrutement au Sud du fleuve Save, qui coupe le Mozambique le long du 22e parallèle. En contrepartie, le Portugal se voit garantir par l'Afrique du Sud un transit important par le port de Lourenço Marques (devenu Maputo après l'indépendance en 1975) et l'acquisition d'or à un prix inférieur à celui du marché international.

En séparant les hommes de leurs familles et en concourant à la monétarisation des campagnes, le système du travail migrant déstructure les sociétés rurales du Sud du Mozambique, devenues dépendantes des revenus des mineurs, des figures enviées qui affaiblissent l'autorité des chefs traditionnels. Ce faisant, il façonne également ces provinces - dont est originaire l'essentiel des migrants mozambicains à Johannesburg - en suscitant un puissant imaginaire de la migration qui pousse, depuis, les hommes de cette région à migrer en Afrique du Sud.

\section{L'apartheid et ses frontières}

La mise en place de l'apartheid après 1948 établit des frontières divisant ceux qui résident sur le sol sud-africain ${ }^{12}$. Comme l'a montré Michel Foucher, il ne s'agit pas seulement d'un ensemble de mesures discriminatoires destinées à assurer la suprématie des populations de seule ascendance européenne, mais aussi d' "une stratégie spatiale, très élaborée et évolutive, et qui s'applique à différents nouveaux scalaires 13 ». Instauré dans une période de forte industrialisation, l'apartheid a notamment pour objectif d'empêcher

11. Crush J., Jeeves A., Yudelman D., South Africa's Labor Empire. A History of Black Migrancy to the Gold Mines, Cape Town, David Philip, 1991 ; Maloka T., "Mines and Labour Migrants in Southern Africa", Journal of Historical Sociology, vol.10, n², 1997, pp. 213-224.

12. Sur la pensée et l'instauration de l'apartheid, voir Posel D., The Making of Apartheid, 19481961. Conflict and Compromise, Oxford, Clarendon Press, 1991.

13. Foucher M., Fronts et frontières. Un tour du monde géopolitique, Paris, Fayard, 1988, p. 215. 
l'installation en ville des migrants ruraux. Le recours aux travailleurs des régions limitrophes de l'Afrique du Sud s'intensifie à cette fin, et ces derniers - des mineurs pour l'essentiel - résident le temps de leur contrat sur le site des installations minières, à l'écart des zones urbaines.

De nouvelles frontières juridiques naissent également. Elles concernent tout d'abord la classification juridique de la population en quatre groupes raciaux : les «Blancs » (Europeans ou Whites), les «Asiatiques 》 (Asians) ou «Indiens » (Indians), les «Africains » (dénommés, selon les époques, Bantus, Africans ou Natives) et les Coloured (habituellement traduit en français par « Métis », quoi que regroupant dans les faits tous ceux qui n'entrent pas dans les trois autres catégories). Cette division en groupes raciaux est complétée par une division des "Africains» en dix sous-groupes, ou «ethnies », et chaque Africain se voit attribuer un groupe ethnique et un territoire où il a vocation à résider. En cela, l'apartheid est, pour reprendre les mots de Foucher, une «ethnogenèse 14 ».

Tout un ensemble de frontières sociales et spatiales résulte de ces frontières juridiques. En 1950, une loi interdit les relations sexuelles entre les Blancs et les membres des autres groupes raciaux. La même année, une autre loi impose des lieux de résidence séparés à chaque groupe racial et, en renforçant la ségrégation déjà existante, modifie la physionomie des villes par des déplacements forcés de populations. En 1951, le Bantu Authorities Act crée, loin des principaux centres urbains, les homelands ou bantoustans, des zones réservées à chaque groupe ethnique africain et censées être leurs «terres d'origine » : il ne laisse que $13 \%$ de la superficie du territoire de l'Afrique du Sud à plus de trois-quarts de sa population, le reste étant réservé aux Blancs, qui n'en représentent qu'à peine plus de $10 \%$.

L'apartheid constitue dans sa finalité un processus de dénationalisation de la population africaine. Entre les années 1960 et le début des années 1980, 3,5 millions d'Africains sont déplacés à cette fin vers les homelands. En 1970, Pretoria retire la nationalité sud-africaine à la population des bantoustans, dont les dirigeants fantoches restent pourtant sous son contrôle. En 1984, les Africains résidant dans les zones «blanches », considérés comme ressortissants d'un homeland, perdent à leur tour la nationalité sud-africaine et doivent demander un permis de séjour sous peine de se trouver en situation illégale. Des centaines de milliers d'Africains qui n'ont jamais vécu dans les zones attribuées à leur groupe ethnique se retrouvent alors étrangers dans leur pays natal.

Dans cette Afrique du Sud de l'apartheid, la situation des Mozambicains, d'après les témoignages que nous avons recueillis de ceux qui l'ont connue, n'est pas foncièrement différente de celle des Noirs sud-africains. Il leur faut 
rester aux marges de la ville blanche, entassés dans les hostels des townships, ces gigantesques blocs sans confort qui abritent les migrants ruraux. Dans les mines, le mode de vie est largement corrélé au lieu de provenance et à l'appartenance ethnique supposée. Les Mozambicains, ordinairement catégorisés "Shangaans », bien qu'ils ne le soient pas, se retrouvent fréquemment dans les mêmes bâtiments que les Shangaans d'Afrique du Sud, dont beaucoup parlent la langue, car le management cherche à diviser les mineurs en les logeant et en gérant leur affectation au travail selon leur origine ethnique ou nationale. Cette politique n'empêche pas la camaraderie entre mineurs, mais les conflits interethniques sont légion dans ces lieux où une forte consommation d'alcool exacerbe les tensions entraînées par la mise en concurrence dans un contexte confiné. L'absence de stabilité et la rotation de travailleurs ne facilitent du reste pas le dépassement des clivages ethniques ${ }^{15}$.

La lutte des mineurs contre l'apartheid voit pourtant s'affirmer des appartenances définies par un critère de nationalité, bien que les identifications ethniques demeurent saillantes. Dans les années 1980, le refus des Mozambicains de participer à certaines grèves leur attire ainsi une réputation de collaborateurs et une image dépréciée qui s'étend parfois à l'ensemble des Shangaans, quelle que soit leur nationalité 16 .

\section{Transformation politique et changements de frontières}

L'indépendance du Mozambique en 1975, la guerre civile qui déchire le pays pendant quinze ans (1977-1992), la fin de l'apartheid en Afrique du Sud et le renforcement de la coopération entre les deux pays sont autant de faits politiques qui ont un effet direct sur les conditions de franchissement de la frontière et les conditions de vie des migrants mozambicains à Johannesburg. L'effacement des frontières de l'apartheid conduit notamment à une nouvelle conception du national et de l'étranger qui alimente l'hostilité des Noirs sudafricains à l'encontre des migrants africains et se traduit par une politique d'immigration restrictive.

\section{De nonveaux flux migratoires}

Après l'indépendance du Mozambique, Pretoria renforce le contrôle de la frontière avec ce voisin dont les nouveaux dirigeants soutiennent les opposants à

15. McNamara J.K., "Brothers and Work Mates: Home Friend Networks in the Social Life of Black Migrants in a Gold Mine Hostel” in Mayer P. (ed.), Black Villagers in an Industrial Society. Anthropological Perspectives on Labour Migration in South Africa, Cape Town, Oxford University Press, 1980, pp. 305-339.

16. Lors des émeutes xénophobes de mai 2008, les Shangaans sud-africains furent ainsi l'objet d'agressions dans les townships de Johannesburg. Voir Tshabalala T., Monako D., "Inside the Mob”, Mail E Guardian, 22 mai 2008. 
l'apartheid. C'est un mur de barbelés qui se construit entre les deux pays. Cela n'empêche cependant pas les Mozambicains de s'employer à la franchir. Quantité de jeunes, principalement originaires de la région de Maputo, continuent en effet de partir travailler en Afrique du Sud comme leurs aînés et, selon les mots de ceux qui l'ont fait à cette époque, ils «sautent la frontière " pour gagner Johannesburg, à moins d'être repris et expulsés, souvent un prélude à une nouvelle tentative de passage. Dans les années 1980, l'intensification de la guerre civile pousse par ailleurs 350000 Mozambicains à fuir leur pays pour l'Afrique du Sud. Le régime de l'apartheid s'en accommode tant qu'ils restent au Nord, dans les zones rurales des bantoustans du Gazankulu et du KaNgwane. Il les traite en revanche en clandestins et les expulse dès qu'ils les quittent ${ }^{17}$. Nombre de ces réfugiés s'installent avec plus ou moins de difficultés dans des villages sudafricains de la même ethnie shangaan ${ }^{18}$. Passer cette frontière est alors une opération à hauts risques. A l'Est du pays, certains meurent de fatigue après des journées de marche ou font des rencontres fatales avec les animaux sauvages du parc national du Kruger qu'ils doivent traverser. Si les frontières du Sud du Mozambique, généralement empruntées pour gagner Johannesburg, sont moins périlleuses, elles sont loin d'être sans dangers, comme en témoigne le nombre de migrants rançonnés en chemin ou abandonnés en route par des passeurs.

C'est pourtant au terme de ces épreuves que, dans la seconde moitié des années 1980, des dizaines de milliers de Mozambicains affluent sur Johannesburg et s'installent dans les townships, les camps de squatters et les quartiers centraux de la métropole délaissés par les populations blanches, apeurées devant la rapidité de la déségrégation. Dès la fin des années 1970 en effet, la ségrégation spatiale commence à s'estomper, et les immeubles de l'inner-city, autrefois exclusivement réservés aux «Blancs», se peuplent d'Indiens sud-africains, d'Africains de Johannesburg, puis de migrants venus des campagnes et d'autres Etats africains ${ }^{19}$.

\section{Des migrants dans la ville}

Si Johannesburg reste aujourd'hui toujours marquée par son découpage racialisé, la fin de l'apartheid en a changé le visage, au point de la rendre méconnaissable à ceux qui l'ont connue en ce temps. La disparition des frontières juridiques qui divisaient la ville en zones raciales a ainsi changé la démographie de la plupart des lieux et accéléré l'insertion des migrants dans son économie. Ceux qui viennent du Mozambique s'emploient principalement

17. Wa Kabwe-Segatti A., «Du rapatriement volontaire au refoulement dissimulé. Les réfugiés mozambicains en Afrique du Sud », Politique africaine, n85, mars 2002, pp. 75-92.

18. Polzer T., “We are all South Africans now': The Integration of Mozambican Refugees in South Africa”, FMSP working paper n8, mai 2004.

19. Sur ce processus, voir Morris A., Bleakness E Light. Inner-City Transition in Hillbrow, Johannesburg, Witwatersrand University Press, 1999. 
dans le secteur informel et, particulièrement, dans ses activités les plus mal payées, comme le bâtiment, la restauration, les ateliers de carrosserie, les salons de coiffure et le commerce de rue. Ils acceptent néanmoins ces emplois facilement, non seulement en raison de leur fréquent faible niveau de qualification, mais aussi parce que, venant d'un pays où les salaires sont encore plus faibles, ils comptent sur leur migration pour épargner de quoi monter le plus vite possible une petite affaire au Mozambique. Leur très fort taux d'activité a été clairement mis en évidence par l'ACP qui montre, de manière pionnière, que la quasi-totalité des hommes mozambicains travaille à Johannesburg, dont plus de la moitié à temps complet, des chiffres de très loin plus élevés que ceux des Noirs sud-africains, des Congolais et des Somaliens, beaucoup moins souvent employés à plein temps et connaissant un chômage qui en touche entre $40 \%$ et $20 \%$. Cette enquête établit en outre que, de ces quatre groupes, ce sont eux qui ont en moyenne les revenus les plus élevés, bien qu'ils restent modestes. Les Noirs sud-africains les méprisent d'ailleurs avant tout pour cette concurrence qu'ils jugent déloyale, et, même s'ils reconnaissent généralement aussi que "ces gars bossent dur ", l'idée qui prévaut parmi eux est bien davantage qu'«ils se contentent de peu» et «volent l'emploi».

Hormis un petit nombre - installé de longue date et constitué de travailleurs qualifiés en situation légale - qui occupe des positions enviables, la plupart des Mozambicains connaissent des conditions de vie très dures. Entassés dans des appartements délabrés du centre ou dans des logements de fortune des townships et de camps de squatters, ils s'épuisent à la tâche, souvent sept jours par semaine, et éprouvent continuellement la menace de l'expulsion et de l'agression. Leur parcours migratoire n'a plus le caractère réglé qu'il possédait du temps où leurs â̂nés travaillaient dans les mines contre une rémunération déterminée à l'avance et pour un temps connu de tous. Partir en Afrique du Sud est désormais placé sous le signe d'une grande incertitude, et leur trajectoire dépend largement d'aléas sur lesquels ils n'ont guère de prise. Quelques-uns, peu nombreux, parviennent bien à épargner suffisamment pour, de retour à Maputo, accéder à la propriété et ouvrir une affaire, généralement une épicerie ou un commerce de détail dans un quartier populaire. Ils réalisent alors l'objectif qu'ils s'étaient fixés à leur départ et, dans un discours satisfait, se montrent intarissables sur l'adversité qu'ils ont su vaincre à Johannesburg, au prix d'un travail acharné, d'économies sou à sou et du renoncement aux plaisirs de l'alcool et des femmes vénales dans lesquels beaucoup de leurs compatriotes auraient englouti leurs gains. La plupart ne connaissent pourtant pas cette fortune, sans nécessairement avoir mené une vie de débauche. Certains oscillent entre Johannesburg et Maputo, gagnant trop peu en Afrique du Sud pour acquérir de quoi envisager une réinstallation définitive. D'autres renoncent à la vie de migrant après la perte d'un emploi, un accident, une agression ou une reconduite à la frontière. Fauchés et penauds, ils reviennent au Mozambique contraints de vivre sous le toit et l'autorité de leur père ou de s'établir dans la grande pauvreté dans des cabanes de roseaux à la périphérie de Maputo, n'ayant pour autre moyen de subsistance que 
des emplois occasionnels et l'aide alimentaire d'églises ou de voisins. Des rudiments d'anglais, le parler du zoulou urbain, ainsi que la connaissance des marchés de Johannesburg et du fonctionnement de la frontière, permet toutefois à certains de ces anciens migrants de gagner quatre sous en faisant du commerce transfrontalier entre l'Afrique du Sud et le Mozambique, dont l'approvisionnement est irrégulier. Les petits profits ainsi dégagés ne leur assurent pas plus qu'à ceux qui ont des emplois peu qualifiés, mais ils leur permettent de se définir positivement comme « faisant des affaires » et d'échapper à la domination pesante d'un employeur. D'autres encore vivotent tant bien que mal à Johannesburg et ne reviennent jamais au Mozambique où, ayant rompu les liens avec leur famille, ils ne disposent plus de point de chute. Cette situation ne concerne d'ailleurs pas que des hommes seuls, mais aussi des familles sans les ressources financières et relationnelles qui rendraient viable un retour à Maputo.

Fait essentiel, la vie des Mozambicains à Johannesburg se caractérise par une recherche de l'invisibilité qui les différencie de ce que l'on observe chez les autres migrants africains et les migrants ruraux sud-africains. Certains d'entre eux - de petits hommes d'affaires ayant généralement acquis la nationalité sudafricaine - prétendent bien s'employer à les organiser, mais leurs efforts ne rencontrent aucune adhésion chez ceux dont ils entendent représenter les intérêts. Il est vrai que leur action concrète consiste moins en un travail politique d'organisation qu'en des activités marchandes consistant, contre rémunération, à servir d'intermédiaires entre les migrants, le consulat du Mozambique et les administrations locales. Cette invisibilité se manifeste encore dans ce que les Mozambicains ne cherchent pas à se regrouper, tel que cela a souvent été décrit à propos des pratiques des immigrés. Ils ne se concentrent ainsi pas dans un espace particulier, comme c'est le cas des Nigérians, des Congolais, des Somaliens et des Ethiopiens. Ils n'occupent également aucune position dominante dans l'économie et ne disposent pas de réseaux leur permettant de peser sur l'accès à un type spécifique d'activité ${ }^{20}$. C'est aussi pour cela qu'ils ne forment pas une niche ou une enclave ethnique, au sens qu'ont ces notions dans les études migratoires, même s'ils sont fortement représentés parmi les coiffeurs, les vendeurs de rue et les ouvriers du bâtiment ${ }^{21}$.

Ce souci de se fondre dans la ville aboutit à ce que, nulle part à Johannesburg, les Mozambicains n'occupent d'espace où leur concentration

20. On ne mentionne ici que les activités légales par manque d'informations fiables sur les différents types de criminalité que l'opinion prête aux Mozambicains, notamment le vol de voitures et le recel de marchandises volées au Mozambique. Les données sur le taux d'incarcération des étrangers n'indiquent pourtant pas une surreprésentation des Mozambicains, et il est bien difficile de savoir si cette donnée tient au caractère infondé des rumeurs les concernant ou à leur discrétion dans ce domaine.

21. Voir notamment à ce sujet: Wilson K., Portes A., "Immigrant Enclaves: An Analysis of the Labor Market Experiences of Cubans in Miami”, American Journal of Sociology, n86, 1980, pp. 295-319; Waldinger R., “The Ethnic Enclave Debate Revisited”, International Journal of Urban and Regional Research, ${ }^{\circ} 17,1993$, pp. 444-452. 
établirait une sorte de frontière de fait par rapport à l'environnement alentour. Au moins trois raisons peuvent être avancées pour l'expliquer. La proximité du Mozambique facilite, en premier lieu, des retours fréquents et rend moins fort le besoin de se regrouper sur la base d'une origine commune que chez les migrants revenant rarement dans leur pays. La parenté linguistique du zoulou et du shangaan permet, en deuxième lieu, d'échapper plus facilement à la traque aux sans-papiers, fréquemment trahis par une absence de maitrise minimale du zoulou parlé à Johannesburg, la lingua franca des Africains de la ville. La fragilité des liens interpersonnels entre Mozambicains constitue, en troisième lieu, un facteur peu propice à l'émergence d'un groupe organisé. Celleci a elle-même des causes distinctes dont les effets se renforcent. Elle réside d'abord dans la pauvreté dans laquelle la majorité vit et contribue à la rupture fréquente d'une relation au moindre conflit sur les dépenses de vie commune. Elle résulte ensuite du manque de confiance des Mozambicains à l'égard d'autrui et de leurs compatriotes, révélé autant par notre enquête de terrain que par les données de l'ACP. Elle procède, enfin, de la faiblesse d'une tradition d'auto-organisation au Mozambique où, quand elles se sont développées après l'indépendance, la plupart des associations de base ont été constituées par des acteurs politiques qui en contrôlent le fonctionnement et dissuadent toute initiative qui échapperait à leurs dirigeants.

\section{Une redéfinition du national et de l'étranger}

L'avènement d'un régime démocratique en Afrique du Sud en 1994 s'accompagne d'une redéfinition des critères du national et de l'étranger. Les anciens bantoustans sont incorporés au territoire national et, alors que seuls les Blancs possédaient une citoyenneté pleine du temps de l'apartheid, tous les Sud-Africains sont proclamés égaux en droit dans une démocratie qui se dit non racialiste 22 . Cela a deux conséquences majeures qui renvoient à l'idée de frontière : d'une part, la « race » ne structure plus désormais ce qui sépare le citoyen du non-citoyen et ne définit donc plus tous les Africains comme des étrangers; d'autre part, et par voie de conséquence, cette nouvelle conception du national érige maintenant une limite juridique entre les Africains de nationalité sud-africaine et ceux originaires d'autres pays du continent. Or, ce changement intervient au moment de l'arrivée massive de migrants étrangers africains dans le pays le plus riche du continent où l'accession de Nelson Mandela à la présidence de la République laisse augurer de grands espoirs. Ils déchantent pourtant vite devant l'hostilité des Noirs sud-africains qui les accusent de l'accroissement de la criminalité, de la propagation du sida et de l'aggravation du chômage alors qu'ils attendent justement du changement politique l'amélioration de leur situation.

22. Sur cette question voir: Chipkin I., Do South Africans Exist? Nationalism, Democracy and the Identity of "The People", Johannesburg, Wits University Press, 2007. 
La référence à la nationalité sud-africaine et aux droits qu'elle confère acquiert, dans ce contexte, une légitimité nouvelle chez ceux qui, marginalisés par l'apartheid, s'estiment lésés par ces migrants. L'hostilité envers ces derniers ne procède bien sûr pas intrinsèquement de la démocratie, mais la nouvelle forme d'appartenance qui en nait sert de justification aux comportements qui l'accompagnent. Des vendeurs de rue étrangers sont régulièrement battus et dépouillés et, dans les townships, des foules en colère s'en prennent aux Mozambicains jusqu'à leur domicile ${ }^{23}$. Leur situation de clandestins en fait aussi des proies de choix pour les policiers sud-africains qui les arrêtent, sauf quand, cas fréquent, ils acceptent de verser un pot-de-vin pour être relâché. Entre 1994, année de l'élection de Mandela, et mars 2005, plus de 1500000 étrangers sont reconduits à la frontière selon les statistiques officielles, parmi lesquels plus de 1150000 ressortissants mozambicains. Les relations économiques entre la République sud-africaine et le Mozambique connaissent pourtant des jours nouveaux à la même époque avec la mise en place du Maputo Development Corridor ${ }^{24}$. La construction d'infrastructures routières, ferroviaires et portuaires dynamise les échanges entre la région de Johannesburg et Maputo, lesquels avaient considérablement diminué depuis l'indépendance du Mozambique. Les années qui suivent immédiatement la fin de l'apartheid voient toutefois un accroissement des expulsions de Mozambicains : de 42350 en 1990 et 71279 en 1994, l'on passe à 131689 en 1995, 157423 en 1996 et 123961 en 1999, avant que les reconduites à la frontière mozambicaine ne se stabilisent entre 80000 et 90000 à partir de 2000. Les trois vagues de régularisation de 1995, 1996 et 1999 ne modifient rien fondamentalement. Ces procédures d'amnistie ne concernent tout d'abord qu'un nombre limité de Mozambicains arrivés en Afrique du Sud avant 1994. Elles ne répondent ensuite pas aux problèmes posés par l'installation de migrants internationaux sur le territoire sud-africain. Elles fonctionnent surtout comme un mécanisme de régulation temporaire de l'immigration clandestine et un instrument de politique étrangère pour le gouvernement issu de l'opposition à l'apartheid qui a une dette morale à l'égard des pays africains ayant soutenu sa lutte. Ce qui frappe, en réalité, est l'étonnante continuité entre le caractère restrictif et répressif des politiques d'immigration avant et après l'arrivée de Mandela à la présidence de la République sud-africaine 25. Une légère inflexion a toutefois eu lieu en 2005 avec la signature d'un accord entre Maputo et Pretoria destiné à faciliter les séjours courts en Afrique du Sud. Ses dispositions participent à la fluidification du commerce transfrontalier et permettent à des migrants de rester dans la légalité pour une durée maxi-

23 . Bouillon A. (dir.), Immigration africaine en Afrique du Sud. Les migrants francophones dans les années 1990, Paris, IFAS-Karthala, 1999; Wa Kabwe-Segatti A., La Place de l'étranger dans la "nouvelle " Afrique du Sud : la réforme d'une politique de l'immigration (1986-2002), thèse pour le doctorat, université de la Réunion / IEP de Bordeaux, 2003.

24 . Söderbaum, F., Taylor, I. (eds.), Regionalism and Uneven Development in Southern Africa. The Case of the Maputo Corridor Development, Aldershot, Ashgate, 2003.

25. Wa Kabwe-Segatti A., op. cit. 
male d'un mois. Son caractère très contraignant en limite cependant la portée à un petit nombre, la majorité des Mozambicains de Johannesburg vivant toujours dans la clandestinité.

\section{Une frontière ethnique comme support de l'identité personnelle}

Les migrants du Mozambique ne réussissent toutefois pas à se rendre toujours invisibles dans Johannesburg. Une majorité y parvient vaille que vaille, après un temps d'adaptation, mais rares sont ceux qui échappent au rappel régulier de leur extranéité et qui se considèrent à l'abri d'une agression. Cette hostilité ambiante les conduit à se penser autrement des autres Africains, en s'en différenciant par la délimitation discursive d'une frontière ethnique sur des critères aussi bien moraux que culturels. Cette dernière section s'intéresse à ce processus qui amène ces migrants à former un groupe ethnique en creux, favorisant le maintien de l'identité personnelle sans pour autant posséder la consistance nécessaire à l'entrée dans une action collective.

\section{Un monde de rapports sociaux racialisés}

Les Mozambicains découvrent à Johannesburg un monde de rapports sociaux racialisés difficiles à imaginer pour qui vient de Maputo, où la fluidité prévaut dans les relations interethniques et où les Africains étrangers rencontrent rarement l'animosité des autochtones. Or, personne n'échappe durablement à une assignation identitaire implacable sitôt franchie la frontière, tant un individu est toujours défini en Afrique du Sud comme membre d'un groupe racial ou ethno-racial et, du moins lors la première interaction, se voit attribuer les traits de personnalité correspondant à la catégorie dans laquelle il a été classé. Cette racialisation des rapports sociaux y possède une force sans commune mesure avec la forme que ce processus prend dans les sociétés qui en viennent peu à peu à se représenter à elles-mêmes comme formées de groupes raciaux en concurrence ${ }^{26}$. Il est bien sûr possible d'échapper en partie ou momentanément à l'identité raciale attribuée, et beaucoup d'échanges sociaux n'auraient pas lieu si les préventions des uns vis-à-vis des autres n'étaient pas suspendues ou retenues dans les interactions de la vie quotidienne. Le préjugé à l'égard de l'autre racial reste néanmoins sous-jacent et, s'il n'intervient pas mécaniquement quand deux individus ont tissé des relations, il revient au galop au moindre différend.

Aussi peu favorable leur soit-il, les migrants mozambicains perpétuent pourtant cet univers racialisé. Car s'ils affirment qu' "au Mozambique, il n'y a pas cette chose des races» ou qu' 'à Maputo, la race d'une personne n'est pas impor-

26. Sur cette question à partir de réflexions du cas français, voir Wieviorka M., « Racisme, racialisation et ethnicisation en France ", Hommes et Migrations, n¹195, février 1996, pp. 27-33, et l'ouvrage coordonné par: Fassin D., Fassin E. (dirs.), De la question sociale à la question raciale ? Représenter la société française, Paris, La Découverte, 2006. 
tante », ils ne procèdent pas autrement à Johannesburg que ses autres habitants. Peu à peu, ils se mettent à déchiffrer la population de la ville à partir des définitions sociales que ce nouvel environnement leur fournit ${ }^{27}$. Leurs propos à ce sujet se caractérisent par leur grande similarité. Les «Blancs» et les «Indiens » ne sont pas en Afrique du Sud, disent-ils, les «mêmes » que ceux qui vivent au Mozambique, et il convient donc de s'en méfier tout particulièrement parce qu' «ilsn'aiment pas les Africains ». A cela, beaucoup ajoutent que «les Africains d'ici sont différents; ils sont racistes avec les autres Africains". L'adoption des catégories locales d'évaluation des étrangers conduit de surcroît les Mozambicains à attribuer aux autres migrants africains les stéréotypes qui les stigmatisent. Ils parlent ainsi communément des Nigérians, des Zimbabwéens et des Congolais comme autant de criminels notoires, c'est-à-dire dans les termes similaires à ceux que les Sud-Africains utilisent habituellement à leur propos.

Entretenir ce type de préjugés ne les distingue pourtant pas de la masse des migrants africains étrangers aux yeux des Noirs sud-africains, largement convaincus de leur supériorité culturelle. Ces derniers les considèrent en effet avant tout comme des Makwerekwere, le terme générique péjoratif qui vaut pour tous les étrangers d'origine africaine. Et quand, dans la conversation, ils se prennent à les différencier, ce qui est rarement le cas, ils ne perçoivent pour la majorité guère de différence entre les «Mozambicans », définis en fonction de leur origine nationale, les « Maputos ", du nom de la ville dont la plupart viennent, et les «Shangaans ", lorsqu'ils parlent cette langue qui est aussi - on l'a vu - celle de l'un des groupes ethniques de l'Afrique du Sud.

\section{Le tracé d'un groupe selon des critères moraux et culturels}

Ces jugements des Mozambicains sur les autres migrants africains de Johannesburg résultent toutefois moins de la simple répétition d'un discours dominant que d'un mode de défense de l'identité personnelle. Ils ne peuvent en effet répondre à la déstabilisation identitaire qu'entraîne la xénophobie qu'en traçant, par le discours qu'ils en viennent à tenir sur autrui et eux-mêmes, une frontière qui dessine en creux un groupe ethnique dans lequel ils se reconnaissent. Ce processus a fréquemment été observé dans les études migratoires. Ainsi, aux Etats-Unis, l'identification des immigrés à un groupe s'est souvent produite après l'émigration, et l'ethnicité qui en a procédé leur a offert une réponse aux besoins d'organisation qu'ils rencontraient dans la société américaine 28. A cet égard, le cas des Mozambicains à Johannesburg n'est pas de prime abord foncièrement différent. Avant la première migration et quand ils arrivent en Afrique du Sud, le fait de venir du même pays n'a pas chez eux grande importance, car ils ne se définissent alors pas fondamentalement par rapport à une ori-

27. Thomas W.I., « Définir la situation » in Grafmeyer Y., Joseph I. (dirs.), L'Ecole de Chicago. Naissance de l'écologie urbaine, Paris, Aubier, 1984 (1 $1^{\mathrm{re}}$ éd. 1923), pp. 79-82.

28 . Poutignat P., Streiff-Fenart J., op. cit. 
gine nationale commune. Tous savent bien qu'ils sont de nationalité mozambicaine, à la différence de ce qu'on observe parfois chez des populations rassemblées à leur insu dans un même ensemble étatique après un nouveau tracé de frontières ou une migration forcée au travers d'étendues non urbanisées. Mais leur capacité à tenir un discours sur le Mozambique et les Mozambicains dépend pour beaucoup de leur ancienneté dans la migration. Ce sont même, à bien des égards, ceux d'entre eux qui vivent depuis le plus longtemps en Afrique du Sud qui différencient avec le plus de netteté les Mozambicains des SudAfricains. Cette conscience ethnique fondée sur la nationalité repose avant tout sur un vécu partagé de la xénophobie à Johannesburg, ce qui, là encore, a déjà été mis en évidence dans d'autres contextes ${ }^{29}$. On retrouve ici, de manière plus générale, les attendus de la contribution séminale de Fredrik Barth à la compréhension de l'ethnicité, à savoir que les groupes ethniques n'existent pas en soi, indépendamment des relations qu'ils entretiennent avec d'autres groupes, mais, au contraire, qu'ils se construisent en définissant leurs frontières dans les interactions qu'ils ont avec ces derniers ${ }^{30}$.

Ce qui fait en revanche la spécificité de ce processus chez les migrants du Mozambique à Johannesburg réside dans trois caractéristiques majeures de leur conscience ethnique. Celle-ci se développe d'abord à partir d'une origine nationale commune sans pourtant qu'ils éprouvent un fort sentiment d'appartenance nationale. Elle ne conduit pas ensuite à une forte ethnicité, mais fonctionne essentiellement comme un support de l'identité personnelle dans un univers où celle-ci est rendue éminemment vulnérable par l'hostilité que leur altérité provoque. Conséquence de ces deux premières caractéristiques et de la recherche d'invisibilité qui prévaut chez ces migrants, elle ne possède pas, enfin, de consistance suffisante pour les conduire à s'organiser, que ce soit en matière de stratégie résidentielle, d'activité économique et de mobilisation politique.

Les Mozambicains tracent cette frontière ethnique en mobilisant trois critères qui renvoient au registre de la morale et à celui de la culture, sans qu'il soit toujours possible de dissocier ces deux dimensions ${ }^{31}$. Le premier est un goût prononcé du labeur qui, selon eux, en fait «les plus travailleurs des Africains». Il revient notamment dans la bouche de ceux qui ont été mineurs et disent l'avoir entendu des contremaîtres blancs. Le deuxième concerne l'honnêteté qui distinguerait les Mozambicains des autres Africains, bien que, rappellent-ils communément, "il y a des personnes mawvaises dans toutes les races». Si ces deux traits se retrouvent peu ou prou chez les travailleurs immigrés du monde

29. Sur le processus de formation d'une conscience ethnique voir : Bastenier A., Qu'est-ce qu'une société ethnique ? Ethnicité et racisme dans les sociétés européennes, Paris, PUF, 2004.

30. Barth F., « Les groupes ethniques et leurs frontières » in Poutignat P., Streiff-Fenart J. (dirs.), Théories de l'ethnicité, Paris, PUF, 1995 (1re éd. 1969), pp. 203-249.

31 . Sur la mise en œuvre de critères moraux dans le traçage de frontières entre groupes et le maintien de sa dignité voir : Lamont M., La Dignité des travailleurs, Paris, Presses de Sciences-Po, 2002. 
entier, le troisième - la dénonciation du tribalisme - est en revanche plus intéressant. Il révèle la transformation en caractéristique culturelle d'un élément du discours politique tenu par le FRELIMO qui, depuis la guerre d'indépendance et après son accession au pouvoir, en appelle au dépassement des appartenances ethniques pour construire la «nation mozambicaine ». Dans cette perspective, le tribalisme évoque à la fois les formes d'appartenance créées par le colonisateur pour diviser les Africains, un ferment de division ensuite encouragé par les puissances étrangères hostiles au jeune Etat indépendant, et le reflet de traditions obscurantistes freinant l'avènement de l'« homme nouveau » dans un pays qui s'est déclaré d'orientation marxiste-léniniste. L'action du FRELIMO n'a pas mis fin, loin s'en faut, aux pratiques ramenées à la tradition (au premier rang desquelles la sorcellerie ${ }^{32}$ ). Afin d'éviter l'accroissement des divisions et de l'hostilité à leur politique, ses dirigeants ont d'ailleurs vite adopté une attitude conciliant «tradition » et «modernité ». Ce discours, largement relayé dans les écoles et les médias de masse, semble en revanche avoir eu pour effet de doter les migrants mozambicains d'un discours qui les fait se considérer plus « avancés » que les Sud-Africains qui en seraient justement encore, selon eux, au temps du tribalisme, dont ils voient la présence dans les tensions qui marquent régulièrement les relations entre Zoulous et Xhosas. C'est là effectivement, bien qu'aucun des Mozambicains rencontrés n'associe directement la dénonciation du tribalisme au discours du FRELIMO, la seule explication à notre sens possible pour rendre compte, chez eux, de l'utilisation de cet argument pour se différencier des Noirs sud-africains.

Le tracé de cette frontière ethnique par référence à la moralité et dénonciation du tribalisme s'articule également autour de l'idée d'une commune humanité de tous les individus dignes de valeur. Les migrants mozambicains jugent pour cette raison que les Noirs sud-africains dérogent à cet idéal, comme en attestent les propos jugés «racistes » par lesquels ils infériorisent les autres Africains au nom d'une prétendue supériorité de leur « race». Appartenance ethnique et appartenance nationale se recouvrent le plus souvent dans cette condamnation du tribalisme et tendent même, de manière plus fondamentale, à s'estomper devant l'affirmation de la commune humanité des individus.

Or, cette conviction de partager, en fin de compte, moins entre eux qu'avec les autres humains entretient la faible ethnicité des Mozambicains à Johannesburg et les prive, de ce fait, d'une ressource susceptible d'étayer une mobilisation fondée sur la participation à un même groupe ethnique. Cela même si les valeurs culturelles et la supériorité morale qu'ils s'attribuent leur offrent des éléments pour maintenir leur identité personnelle, en rejetant le stigmate d'infériorité dont les Noirs sud-africains les frappent.

32. Voir notamment à ce sujet : Israel P., « Déchirures et rumeurs. La chasse au sorcier et l'héritage idéologique de la révolution socialiste au Mozambique (Muidumbe, 2002-2003)», Cabiers d'études africaines, dossier « Territoires sorciers », n¹89-190, 2008, pp. 209-236. 


\section{Note finale}

Le rôle des frontières étatiques dans la vie des migrants, on le sait, est loin de faire consensus. Un courant de recherches insiste sur leur place croissante dans le contrôle des déplacements ${ }^{33}$. Une autre perspective souligne différemment la capacité des individus à se dégager de ces contraintes pour vivre dans des espaces transnationaux ${ }^{34}$. Le cas des Mozambicains dans le Johannesburg post-apartheid nuance considérablement la portée de cette seconde approche, sans pour autant s'inscrire complètement dans la première. Ces migrants parviennent en effet à franchir aujourd'hui la frontière sud-africaine relativement aisément sans que, pour autant, des frontières cessent de caractériser leur vie en migration.

Il faut, pour l'apprécier pleinement, ne pas se limiter à considérer le seul passage des frontières des Etats. L'accès à la citoyenneté trace, d'une part, une frontière entre ceux qui la possèdent et ceux qui n'en jouissent pas. Aussi trivial soit ce rappel, ce double processus d'inclusion et d'exclusion gagne néanmoins à être compris en fonction du contexte sociopolitique où il intervient. Dans l'Afrique du Sud contemporaine, il a notamment eu pour conséquence de dresser une frontière entre les Noirs sud-africains et les Africains étrangers et a donné aux premiers, privés du statut de citoyen du temps de l'apartheid, un argument justifiant leur hostilité à l'égard des seconds. La réponse que les migrants du Mozambique apportent à cette violence repose, par ailleurs, sur la construction d'une frontière d'un autre ordre. Face à ce qui est vécu comme une épreuve, ceux-ci se découvrent, on l'a vu, une origine et des valeurs communes, ce sans quoi il n'y a pas de groupe ethnique.

La recherche de l'invisibilité dans la ville et la préférence pour des stratégies individuelles entravent toutefois chez eux toute dynamique d'organisation fondée sur un élément ethnique ou national. Aucune pratique collective ne résulte donc de la formation d'une conscience ethnique et de l'identification à un groupe national, hormis celle qui consiste à entretenir et à durcir, dans leurs considérations sur les autres Africains, la frontière ethnique d'un groupe créé par le vécu de l'immigration.

33. Voir, entre autres, à ce propos: Castles S., Globalization and Ethnicity. From Migrant Workers to Transnational Citizens, London, Sage, 2000 ; Blom Hansen T., Stepputat F. (eds.), Sovereign Bodies. Citizens, Migrants and States in the Postcolonial World, Princeton, Princeton University Press, 2005 ; Kobelinsky C., Makaremi C. (dirs.), « Confinement des étrangers : entre circulation et enfermement », Cultures $\mathcal{E}$ Conflits, ${ }^{\circ} 71$, automne 2008.

34 . Pour se faire une idée de la diversité des thèmes qui peuvent être considérés comme procédant de cette approche, voir le recueil de textes coordonné par Khagram S., Levitt P. (eds.), The Transnational Studies Reader. Intersections and Innovations, New York / London, Routledge, 2008. 
La présence de la frontière dans leur rapport à autrui n'est alors jamais aussi prononcée que dans ces différentes logiques d'action. Car celles-ci les amènent en même temps à chercher à se confondre avec l'environnement urbain, en raison des risques qu'entrâne potentiellement leur visibilisation, et à se définir comme un groupe caractérisé par des attributs valorisables, afin d'établir un rapport positif à soi dans un monde hostile et déstabilisant pour l'identité personnelle. 